\title{
Histopathological and tumor characteristics associated with false negative axillary ultrasonography results in breast cancer
}

\author{
Merve Gursoy¹, Gulten Sezgin², Emine Merve Horoz ${ }^{2}$, Berna Dirim Mete ${ }^{1}$, Nezahat Erdogan²
}

${ }^{1}$ Izmir Democracy University, Faculty of Medicine, Department of Radiology, ${ }^{2}$ Izmir Katip Celebi University Ataturk Training and Research Hospital, Department of Radiology, Izmir, Turkey

\begin{abstract}
Aims: As the false-negative rates of axillary ultrasonography (AUS) (21\%-48\%) are not optimal, the demand for axillary surgery remains even if AUS is negative. The aim of this study is to determine the histopathological and tumor characteristics associated with false-negative AUS results. Materials and methods: Patients with normal AUS were divided into two groups as true-negative and false-negative according to the histopathology results of axillary lymph nodes. Two groups were compared in terms of age, histological grade of the primary tumor, histological size of the primary tumor, histological type, lymphovascular invasion (LVI), and ultrasonography BI-RADS classification of the primary tumor. The number of metastatic lymph nodes, size of the largest metastatic lymph node and the number of micrometastatic lymph nodes were also noted in the false-negative group. Results: There were 152 patients with normal preoperative AUS in the study group. The false-negative AUS rate was $20.4 \%$. The incidence of invasive lobular carcinoma (ILC) and the mean tumor size was significantly greater in the false-negative group. Micrometastasis was present in 3 patients $(3 / 31,9.6 \%)$, the mean of the largest metastatic lymph nodes was $12.5 \mathrm{~mm}$, the mean total number of malignant lymph nodes was 1.9 in the false-negative group. In $25 / 31$ (80.6\%) of the patients, there were less than or equal to 2 metastatic lymph nodes. The presence of LVI was higher in the false-negative group. There was no significant difference between the groups in terms of the other parameters. Conclusion: Before stating that the axilla is normal on ultrasonography, a careful evaluation should be made in patients with a mass $>2 \mathrm{~cm}$ in size and/ or ILC diagnosis.
\end{abstract}

Keywords: ultrasound; axilla; breast cancer; false negative

\section{Introduction}

For the correct staging of breast cancer and the implementation of appropriate treatment, o knowing the status of axillary lymph nodes is mandatory. In the determination of lymph node involvement, physical examina-

Received 10.01.2019 Accepted 22.03.2019

Med Ultrason

2019, Vol. 21, No 3, 232-238

Corresponding author: Merve Gursoy, MD

Izmir Democracy University, Faculty of

Medicine, Department of Radiology,

Mithatpaşa Cad. Aksel Gursel Bul. No:14

Karabağlar Izmir/Turkey

Phone: +90 5062920046

Fax: +90 2322601004

E-mail: gursoymerve@yahoo.com tion alone is not sufficient. Preoperative axillary ultrasonography (AUS) with the sensitivity of $50-70 \%$ and specificity of $87-95 \%$ is an indispensable part of staging [1]. When AUS is positive, sentinel lymph node biopsy (SLNB), which is an invasive procedure and requires additional costs, is passed and axillary lymph node dissection (ALND) can be applied [2-4]. Thus. time and expense can be saved. However, as the false-negative rates of AUS (21\%-48\%) are not optimal, the demand for axillary surgery remains even if AUS is negative [58]. Therefore, in many centers AUS is combined with fine needle aspiration biopsy (FNAB) and the accuracy is increased in the determination of metastatic axillary lymph nodes [9-11]. However, a negative FNAB still does not remove the necessity for SLNB. All this classic information started to change with the recent Z0011 Trial 
designed by the American College of Surgeons Oncology Group (ACOSOG). In the Z0011 trial, there was shown to be no advantage of the completion of ALND in the presence of positive SLNB. This means that more extensive surgery of the axilla does not provide an additional survival benefit or change the prognosis [12].

These developments have raised new questions. In patients with no lymph node found clinically, when a lymph node is not determined on AUS, can all axillary surgery be abandoned? This question was attempted to answer in the Sentinel versus Observation after Axillary Ultrasound (SOUND) trial [13]. In that prospective randomized controlled trial, patients with breast cancer $\leq 2 \mathrm{~cm}$ without a positive lymph node were randomly separated into 2 groups, one applied with SLNB, and the other with no axillary surgery. This study is ongoing so results are still awaited of the comparisons of the groups in respect of axillary recurrence and disease-free survival.

In the near future, axillary surgery may be able to be completely eliminated in patients not determined with lymph node on AUS. All these reasons increase the importance of the false-negative rates of AUS.

The aim of this study was to determine the histopathological and tumor characteristics associated with false-negative AUS results.

\section{Materials and methods}

\section{Patients}

This retrospective study was approved by the Institutional Review Board of our hospital. Since the study was retrospective, informed consent by patients and providers was not required.

$\mathrm{T} 1$ or T2 breast cancer patients without clinically lymph node who had normal AUS between 2014 January and 2018 January were reviewed retrospectively. Male patients, patients with incomplete medical records, recurrent breast cancer, distant metastasis and previous neoadjuvant chemotherapy were excluded from the study. Patients without AUS before surgery were also excluded.

\section{Pre-operative AUS and SLNB}

A breast radiologist, with 10 years of experience, performed all AUS interpretations (Hitachi HI Vision Preirus Machine, Hitachi Inc., Japan). The lymph nodes were accepted as "metastatic" when the cortex-medulla differentiation was absent, cortical thickness was greater than $2 \mathrm{~mm}$, cortex was hypoechoic, longitudinal to transverse ratio was less than 2 or short axis was greater than 10 $\mathrm{mm}$, and the hilum of the lymph node was compressed or replaced completely [14-15]. Patients without positive AUS underwent SLNB or ALND of at least four nodes when the isotope was absent. Dual blue dye and radioisotope localization was used in SLNB. Due to the unavailability of the isotope, ALND without SLNB was applied in 3 patients in the false-negative group and in 9 patients in the true-negative group. Patients with metastatic lymph node greater than $2 \mathrm{~mm}$ in SLNB underwent ALND. Lymph node histology status was the standard reference for this study. Patients were categorized as true-negative and false-negative according to the histopathology results of the axillary lymph nodes.

The histological grades of the primary tumor were divided into 2 groups as low grade and high grade. Grade 1 tumors were accepted as low grade and grade 2 and 3 tumors were accepted as high grade. The histological type of the primary tumor was divided into 3 groups as invasive ductal carcinoma (IDC), invasive lobular carcinoma (ILC) and others (medullary, apocrine, etc). The largest tumor diameter was accepted as the histological size of the primary tumor and categorized as less than or equal to 20 $\mathrm{mm}(\leq 20 \mathrm{~mm})$ and larger than $20 \mathrm{~mm}(>20 \mathrm{~mm})$. Ultrasonography Breast Imaging Reporting and Data Systems (BI-RADS) classification of the primary tumor was also evaluated and categorized as BI-RADS 4 and 5. The biological subtypes of the primary tumor were divided into 4 categories as luminal-A, luminal-B, human epidermal growth factor receptor 2 (HER2) enriched and triple-negative [16]. Luminal-A subtype express estrogen receptor (ER) and/or progesterone receptor (PR) with negative HER2 and low Ki67 index ( $<15 \%)$, luminal-B subtype is identified as ER-positive, HER2-negative and high Ki67 index $(>15 \%)$ or ER-positive, HER2-positive and any Ki67 index, HER2 enriched is identified as ER-negative, PR-negative and HER2-positive, triple negative subtype is identified as ER-negative, PR-negative and HER2negative. Lymphovascular invasion (LVI) was also evaluated and categorized as present or absent.

We compared two groups (true-negative and falsenegative) in terms of age, histological grade of the primary tumor (low grade, high grade), histological size of the primary tumor $(\leq 20 \mathrm{~mm},>20 \mathrm{~mm}$ ), histological type (IDC, ILC, others), the presence or absence of LVI and ultrasonography BI-RADS classification of the primary tumor (BI-RADS 4,5).

The number of metastatic lymph nodes, size of the largest metastatic lymph node and the number of micrometastatic lymph nodes were also noted in the false-negative group. The largest nodal metastasis diameter was accepted as the lymph node size.

\section{Statistical analysis}

Data analyses were performed using SPSS, version 22.0. The independent sample t-test was used to compare the age distribution between the groups. The frequency distributions of histological size, histological type and 
ultrasonography BI-RADS classification of the primary tumor in the two groups were examined using Pearson chi-squared analysis. The frequency distributions of histological grade, the biological subtype of the primary tumor and presence of LVI were examined using Fisher's exact test. Variables with a $p$ value of $<0.1$ on univariate analysis were entered into a backward stepwise multiple logistic regression to ensure the inclusion of important variables into the multivariate regression model. Variables with resulting $p$ values of $>0.1$ were then eliminated from the regression model. Using this method, only the significant predictors for false-negative AUS remained in the model. The effect size was quantified by odds ratios with $95 \%$ confidence intervals. The level of significance was set at 0.05 .

\section{Results}

The study group consisted of 152 patients with normal preoperative AUS. According to the last pathological evaluation, 121/152 patients were not determined with a metastatic lymph node and the true-negative rate of AUS was $79.6 \%$. In 31 patients, the pathological lymph node was determined after surgery and the false-negative AUS rate was $20.4 \%$. In the false-negative group, micrometastasis was present in 3 patients $(3 / 31,9.6 \%)$, the mean of the largest metastatic lymph nodes was $12.5 \mathrm{~mm}$ (range,
Table I. Histopathological features of axillary lymph nodes in the false-negative group

\begin{tabular}{lll}
\hline & & $\begin{array}{l}\text { False-negative } \\
\text { group }\end{array}$ \\
& & n (\%) \\
\hline Number of positive & $\leq 2$ & $25(80.6)$ \\
lymph nodes & $>2$ & $6(19.4)$ \\
Size of the largest & Micrometastatic & $3(9.7)$ \\
metastatic & $\leq 10$ & $13(42)$ \\
lymph node & $10-20$ & $9(29)$ \\
(mm) & $\geq 20$ & $6(19.3)$ \\
Total & & $\mathbf{3 1}$ \\
\hline
\end{tabular}

$\mathrm{n}$ : Number of patients

6-25 $\mathrm{mm}$ ), the mean total number of removed lymph nodes was 3.4 in SLNB or ALND if the isotope was unavailable and 9.0 in SLNB with completion of ALND or ALND alone. The average of the malignant lymph node count was 1.9 (range, 1-5). In 25/31 (80.6\%) of the patients, there were less than or equal to 2 metastatic lymph nodes (Table I).

The mean age was $58.4 \pm 13.23$ years (range, 19-89 years) in the true negative group and $54.6 \pm 13.11$ years (range, 33-82 years) in the false-negative group. There was no significant difference in the age distribution of the two groups $(\mathrm{p}=0.162)$. There was no statistically significant difference between the groups in respect of histological grade, biological subtypes of the primary tumor and

Table II. Tumor characteristics and histopathological factors related to axillary ultrasonography results

\begin{tabular}{|c|c|c|c|c|}
\hline & & & ups & \\
\hline & & $\begin{array}{l}\text { True-negative } \\
\text { n\% }\end{array}$ & $\begin{array}{l}\text { False-negative } \\
\text { n\% }\end{array}$ & $\mathbf{p}$ \\
\hline Grade & Low & $20(16.5)$ & $2(6.5)$ & $0.251 *$ \\
\hline & High & $101(83.5)$ & $29(93.5)$ & \\
\hline BI-RADS & BI-RADS 4 & $55(45.5)$ & $13(41.9)$ & $0.725 * *$ \\
\hline & BI-RADS 5 & $66(54.5)$ & $18(58.1)$ & \\
\hline Histological type & IDC & $82(67.8)$ & $15(48.4)$ & $0.006 * *$ \\
\hline & ILC & 14(11.6) & $11(35.5)$ & \\
\hline & Others & $25(20.7)$ & $5(16.1)$ & \\
\hline Size of the primary tumor & $\leq 20 \mathrm{~mm}$ & $74(61.2)$ & $9(29)$ & $0.001 * *$ \\
\hline & $>20 \mathrm{~mm}$ & $47(38.8)$ & $22(71)$ & \\
\hline Average (mm) & & 18.4 & 31 & \\
\hline Biological subtypes & Luminal-A & $64(52.9)$ & $20(64.6)$ & $0.778 *$ \\
\hline & Luminal-B & $42(34.7)$ & $9(29)$ & \\
\hline & HER2 Enriched & $6(5)$ & $1(3.2)$ & \\
\hline & $\mathrm{TN}$ & $9(7.4)$ & $1(3.2)$ & \\
\hline LVI & Yes & $10(8.3)$ & $7(22.6)$ & $0.048 *$ \\
\hline & No & $111(91.7)$ & $24(77.4)$ & \\
\hline Total & & $121(79.6)$ & $31(20.4)$ & - \\
\hline
\end{tabular}

* Fisher's Exact test, ** Pearson Chi-Square; TN: Triple-negative; n: Number of patients, LVI: lymphovascular invasion 
Med Ultrason 2019; 21(3): 232-238

ultrasonography BI-RADS classification of the primary tumor ( $\mathrm{p}>0.05)$ (Table II).

There was a significant difference between the groups in terms of the histological type of the primary tumor $(p=0.006)$. ILC subtype was seen with significantly higher incidence in the false-negative group. Then, paired comparisons were made of ILC with other histological types in the true-negative and false-negative groups (Table III). ILC was seen at statistically significantly higher rates in the false-negative group than IDC $(\mathrm{p}=0.002)$ and other subtypes $(\mathrm{p}=0.026)$. The average of malignant lymph node count was 2.3 in patients with ILC, 1.8 in patients with IDC and 1.4 in those with other types.

Histological size of the primary tumor differed significantly between the groups $(p=0.001)$. The mean tumor size was significantly greater in the false-negative group $(31.0 \mathrm{~mm})$ than the true-negative group $(18.4 \mathrm{~mm})$. The tumor size was still a statistically significant factor in the false-negative group after controlling for lobular histology. The mean tumor size of invasive lobular carcinoma patients was $23.3 \mathrm{~mm}$ in the true-negative group and 30.0 $\mathrm{mm}$ in the false-negative group. The mean histological size of the primary tumor was statistically significantly greater in the false-negative group with ILC histological type than in the true-negative group with ILC histological type $(\mathrm{p}=0.011)$.

The presence of LVI was seen at a higher rate in the false-negative group $(7 / 31,22.6 \%)$ than the true-negative group (10/121, 8.3\%). But, LVI did not show statistical significance on multivariate logistic regression analysis $(\mathrm{p}=0.069)$.

The multivariate analysis is outlined in Table IV. Histological type and histological size of the primary tumor showed statistically significance on multivariate logistic regression analysis $(\mathrm{p}=0.021, \mathrm{p}=0.002$ respectively).

\section{Discussions}

The results of prospective randomized studies conducted in recent years have created changes in the view of axillary surgery. In the Z0011 study, patients with 1-2 positive lymph nodes on SLNB were randomly separated into 2 groups as those applied or not with ALND. The results of the study showed no significant difference between the 2 groups (SLNB alone or SLNB+ALND) in respect of locoregional recurrence [12,17]. In the study performed by Rudenstam et al [18], clinically node-negative patients with primary mass less than $5 \mathrm{~cm}$ were included. As a result of SLNB, patients determined with 1 or more micrometastatic $(\leq 2 \mathrm{~mm})$ axillary lymph nodes were randomized as those applied or not with ALND. There was no significant difference between the groups
Table III. Dual comparisons of histological types

\begin{tabular}{llllll}
\hline & & \multicolumn{2}{c}{ Groups } & & \\
\cline { 3 - 5 } & & $\begin{array}{l}\text { True- } \\
\text { negative } \\
\text { n\% }\end{array}$ & $\begin{array}{l}\text { False- } \\
\text { negative } \\
\mathbf{n \%}\end{array}$ & Total & $\mathbf{p} \%$ \\
\hline Histo- & IDC & $82(67.8)$ & $15(48.4)$ & $97(63.8)$ & $\mathbf{0 . 0 0 2}$ \\
logical & ILC & $14(11.6)$ & $11(35.5)$ & $25(16.4)$ & \\
type & IDC & $82(67.8)$ & $15(48.4)$ & $97(63.8)$ & 1.000 \\
& Others & $25(20.7)$ & $5(16.1)$ & $30(19.7)$ & \\
& ILC & $14(11.6)$ & $11(35.5)$ & $25(16.4)$ & $\mathbf{0 . 0 2 6}$ \\
& Others & $25(20.7)$ & $5(16.1)$ & $30(19.7)$ & \\
\hline
\end{tabular}

IDC: Invasive ductal carcinoma, ILC: Invasive lobular carcinoma, $\mathrm{n}$ : Number of patients

Table IV. Multivariable associations between histopathological/tumor characteristics and false-negative results

\begin{tabular}{llll}
\hline & \multicolumn{3}{c}{$\begin{array}{c}\text { Multivariate logistic regression } \\
\text { analysis }\end{array}$} \\
\cline { 2 - 4 } & Odds ratio & $95 \% \mathrm{CI}$ & $\mathrm{p}$ value \\
\hline $\begin{array}{l}\text { ILC histological } \\
\text { subtype }\end{array}$ & 4.79 & $1.26-18.23$ & $\mathbf{0 . 0 2 1}$ \\
$\begin{array}{l}\text { Histological size of } \\
\text { the primary tumor }\end{array}$ & 4.30 & $1.72-10.77$ & $\mathbf{0 . 0 0 2}$ \\
LVI & 3.02 & $0.91-9.95$ & 0.069 \\
\hline
\end{tabular}

IDC: Invasive ductal carcinoma, ILC: Invasive lobular carcinoma, LVI: Lymphovascular invasion, CI: Confidence interval

in respect of local recurrence and disease-free survival events [18]. These studies demonstrated that despite SLNB positivity in early breast cancer patients, ALND could be skipped. These changes alter the role of preoperative AUS in axillary surgery in breast cancer patients. In the SOUND trial conducted in Europe, AUS negative patients were separated into 2 groups [13]. One group was applied with SLNB and no axillary surgery was applied to the other group and the groups were compared in respect of axillary recurrence and survival. This study is still ongoing. If no significant difference emerges between the groups, as expected, a debate could start in the near future about the replacement of SLNB with AUS. When it is considered that a normal AUS result discounts axillary surgery, the importance of false-negative results increases. When no axillary surgery is applied because of a false-negative AUS result, metastatic lymph nodes could be left in the axilla and nodal involvement may not be determined, so the adjuvant treatment plan (chemohormonal therapy and radiotherapy) implemented will be not at the optimal level. In our study, the false-negative AUS rate was $20.4 \%$. Although there are similar results in literature to those of our study, the false-negative range is wide $(21 \%-48 \%)$ [6-8]. As US is operator-dependent, the experience of the operator could contribute to the dif- 
ference in these rates. This was not an issue in the current study as the AUS was applied by a single individual with 10 years of experience.

Another factor, which could have an effect on falsenegative results, is the presence of micrometastases. Nwaogu et al [6] determined micrometastasis at the rate of $48 \%$ in patients with false-negative AUS. Swinson et al [19] demonstrated that axillary US and FNAB have higher sensitivity and specificity in patients with macrometastatic lymph nodes than in those with micrometastatic nodes, but no patient was identified with micrometastatic nodal involvement in that series. When it is considered that micrometastasis does not change the lymph node architecture to a degree that will make a US difference, the skipping of AUS can be expected. In the current study, micrometastasis was present in only 3 patients $(3 / 31,9.6 \%)$ in the false-negative group.

There were $\leq 2$ metastatic lymph nodes in $25(25 / 31$, $80.6 \%$ ) patients in the false-negative group of the current study. In the study by Nwaogu et al [6] this rate was $88 \%$. Thus, the vast majority of false-negative AUS patients have minimal axillary involvement. Nath et al [20] revealed that in patients with metastatic lymph node in SLNB but with normal preoperative AUS only $8.7 \%$ had an additional metastatic lymph node after ALND. This means that SLNB in patients with normal AUS, is adequate for cleaning the axilla and ALND does not provide additional benefit in the majority of patients. Thus, normal preoperative AUS can predict not only minimal axilla involvement but also limit the axillary surgery with SLNB, which is a less invasive method.

The frequency of ILC was significantly higher in the false-negative group than IDC and the other histological subtypes. Neal et al [21] reported that while $4 \%$ of axillary metastases were misdiagnosed on AUS in IDC, 17\% of metastases were missed in ILC. Similarly, Hackney et al [22] demonstrated that false-negative results were more common in ILC. That ILC higher false-negative rates was an anticipated result in the current study. Histologically, ILC is formed of single rows of small, uniform cells with narrow cytoplasm. This microscopic nature of the tumor causes growth by protecting the normal anatomy without forming a sharp-bordered mass. This provides more subtle findings on imaging. Moreover, in the current study, the mean number of malignant lymph nodes in patients with ILC was greater than in those with IDC or other types [2.3, 1.8 and 1.4, respectively). Reyna et al [7] also demonstrated a lower pathological burden of axillary disease $(<2$ total positive nodes $)$ in IDC than in non-ductal carcinomas after a false-negative evaluation.

There was a statistically significant difference between the true-negative and the false-negative groups in the current study in respect of the histological size of the primary tumor (mean size was $31 \mathrm{~mm}$ in the falsenegative group and $18.4 \mathrm{~mm}$ in the true-negative group). Gipponi et al [5] revealed that the negative predictive value of AUS and FNAB were higher in T1 tumors than T2 tumors $(85.5 \%, 58.9 \%$, respectively). They related this result to the higher metastatic potential of advanced breast cancers. It is known that larger dimension tumors create more axillary involvement compared to small tumors. Furthermore, the sensitivity of AUS is correlated to the histological size of the primary tumor and the number of involved axillary lymph nodes [23]. Therefore, our finding that the histological size of the primary tumor was smaller in the true-negative group and, associated with that, there was less axillary involvement, was an expected result. Similarly, Johnson et al [24] in their study, which focused on tumor pathological features associated with false-negative AUS results, found the tumor size to be larger in the false-negative group. They stated that AUS may miss the small axillary metastases.

The presence of LVI was higher in the false-negative group. But, LVI did not show statistically significance on multivariate logistic regression analysis. Johnson et al stated that a false-negative AUS was more likely in tumors with LVI because of the higher pretest probability of metastatic disease [24].

In respect of the histological grade of the primary tumor, it was expected that there would be more highgrade tumors in the false-negative group because of the growth potential. High grade was determined at the rate of $93.5 \%$ in the false-negative group and at $83.5 \%$ in the true-negative group, but the difference did not meet statistical significance.

When the BI-RADS classification was examined, there was greater suspicion of malignancy in patients with BI-RADS 5 primary tumor and it was predicted that there would be greater axillary involvement associated with this. It was therefore thought that there would be more BI-RADS 5 patients in the false-negative group. BI-RADS 5 was determined at the rate of $58.1 \%$ in the false-negative group and at $54.5 \%$ in the true-negative group but the difference failed to meet statistical significance. Similarly, no difference was determined in respect of the biological subtypes of the primary tumor. Johnson et al [24] and Moorman et al [25] demonstrated that tumors which express ER or PR had a higher possibility to have false-negative AUS. The association between false-negative AUS result and ER expression is uncertain. PR positive tumors have a higher potential for axillary metastases and this situation may be related with false-negative AUS results. In our study the subgroups of patients according to tumor molecular biology were 
relatively small and there is a need for studies with larger series to reach such a finding.

The limitations of this study are related in principal to the retrospective design and the small sample size. But, there are few studies in the literature that have focused only on the false-negative results in AUS. There is a real need for further prospective studies with a larger series on this subject.

In the majority of the published studies preoperative AUS was applied by different operators. This situation may cause inconsistency among the results. The strong point of our study is that AUS was applied by a single operator with 10 years of experience. This had a significant effect for the generalizability of the findings.

Opinions of axillary surgery have started to change as a result of studies conducted in recent years. Rather than extensive surgery in early stage breast cancer patients, limited lymph node dissection has come to the fore. It may even be that in the near future, axillary surgery (SLNB or ALND) will be able to be eliminated for patients with normal AUS. This increases the importance of false-negative results. By determining the potential factors that could cause a false-negative result on AUS, it could be possible to determine potential metastatic lymph nodes with a more detailed evaluation in these areas.

In conclusion, higher false-negative AUS rates can be found in larger masses and in the ILC histological subtype. Therefore, before stating that the axilla is normal on US, a careful evaluation should be made in patients with a mass $>2 \mathrm{~cm}$ and/or ILC diagnosis. US is an operatordependent modality and the results vary among radiologists and institutions but these causes of errors can be removed or minimized by stating definitive criteria for the interpretation of AUS findings. It is very important to achieve higher inter- and intraobserver agreement. Nevertheless, ILC subtype has a privileged position because of the high false-negative rates. Even when visible lymph nodes are present in the axilla in patients with ILC, a biopsy should be performed irrespective of the imaging characteristics.

\section{Conflict of interest: none}

\section{References}

1. Alvarez S, Anorbe E, Alcorta P, Lopez F, Alonso I, Cortes J. Role of sonography in the diagnosis of axillary lymph node metastases in breast cancer: a systematic review. AJR Am J Roentgenol 2006;186:1342-1348.

2. Deurloo EE, Tanis PJ, Gilhuijs KG, et al. Reduction in the number of sentinel lymph node procedures by preoperative ultrasonography of the axilla in breast cancer. Eur J Cancer 2003;39:1068-1073.
3. van Rijk MC, Deurloo EE, Nieweg OE, et al. Ultrasonography and fine-needle aspiration cytology can spare breast cancer patients unnecessary sentinel lymph node biopsy. Ann Surg Oncol 2006;13:31-35.

4. Tahir M, Osman KA, Shabbir J, et al. Preoperative axillary staging in breast cancer-saving time and resources. Breast J 2008;14:369-371.

5. Gipponi M, Fregatti P, Garlaschi A, et al. Axillary ultrasound and Fine-Needle Aspiration Cytology in the preoperative staging of axillary node metastasis in breast cancer patients. Breast 2016;30:146-150.

6. Nwaogu IY, Yan Y, Appleton CM, Cyr AE, Margenthaler JA. Predictors of false negative axillary ultrasound in breast cancer. J Surg Res 2015;198:351-354.

7. Reyna C, Lee MC, Frelick A, Khakpour N, Laronga C, Kiluk JV. Axillary burden of disease following false-negative preoperative axillary evaluation. Am J Surg 2014;208:577-581.

8. Yang WT, Ahuja A, Tang A, Suen M, King W, Metreweli C. High resolution sonographic detection of axillary lymph node metastases in breast cancer. J Ultrasound Med 1996;15:241-246.

9. Boughey JC, Moriarty JP, Degnim AC, Gregg MS, Egginton JS, Long KH. Cost modeling of preoperative axillary ultrasound and fine-needle aspiration to guide surgery for invasive breast cancer. Ann Surg Oncol 2010;17:953-958.

10. Boughey JC, Middleton LP, Harker L, et al. Utility of ultrasound and fine-needle aspiration biopsy of the axilla in the assessment of invasive lobular carcinoma of the breast. Am J Surg 2007;194:450-455.

11. Cowher MS, Erb KM, Poller W, Julian TB. Correlation of the use of axillary ultrasound and lymph node needle biopsy with surgical lymph node pathology in patients with invasive breast cancer. Am J Surg 2008;196:756-759.

12. Giuliano AE, Ballman K, McCall L, et al. Locoregional recurrence after sentinel lymph node dissection with or without axillary dissection in patients with sentinel lymph node metastases: Long-term follow-up from the American College of Surgeons Oncology Group (Alliance) ACOSOG Z0011 Randomized Trial. Ann Surg 2016;264:413-420.

13. Gentilini O, Veronesi U. Abandoning sentinel lymph node biopsy in early breast cancer? A new trial in progress at the European Institute of Oncology of Milan (SOUND: Sentinel node vs Observation after axillary UltraSouND). Breast 2012;21:678-681.

14. Choi YJ, Ko EY, Han BK, Shin JH, Kang SS, Hahn SY. High-resolution ultrasonographic features of axillary lymph node metastasis in patients with breast cancer. Breast 2009;18:119-122.

15. Bedi DG, Krishnamurthy R, Krishnamurthy S, et al. Cortical morphologic features of axillary lymph nodes as a predictor of metastasis in breast cancer: in vitro sonographic study. AJR Am J Roentgenol 2008;191:646-652.

16. Sørlie T, Perou CM, Tibshirani R, et al. Gene expression patterns of breast carcinomas distinguish tumor subclasses with clinical implications. Proc Natl Acad Sci U S A 2001;98:10869-10874. 
17. Giuliano AE, McCall L, Beitsch P, et al. Locoregional recurrence after sentinel lymph node dissection with or without axillary dissection in patients with sentinel lymph node metastases: the American College of Surgeons Oncology Group Z0011 randomized trial. Ann Surg 2010;252:426432.

18. International Breast Cancer Study Group, Rudenstam CM, Zahrieh D, Forbes JF, et al. Randomized trial comparing axillary clearance versus no axillary clearance in older patients with breast cancer: first results of International Breast Cancer Study Group Trial 10-93. J Clin Oncol 2006;24:337-344.

19. Swinson C, Ravichandran D, Nayagam M, Allen S. Ultrasound and fine needle aspiration cytology of the axilla in the pre-operative identification of axillary nodal involvement in breast cancer. Eur J Surg Oncol 2009;35:1152-1157.

20. Nath J, Sami N, Massey J, Donnelly J, Corder AP. Selection for axillary clearance in breast cancer (ultrasound negative, sentinel node positive patients have low rates of further metastases). Eur J Surg Oncol 2013;39:450-454.
21. Neal CH, Daly CP, Nees AV, Helvie MA. Can preoperative axillary US help exclude N2 and N3 metastatic breast cancer? Radiology 2010;257:335-341.

22. Hackney L, Williams S, Bajwa S, Morley-Davies AJ, Kirby RM, Britton I. Influence of tumor histology on preoperative staging accuracy of breast metastases to the axilla. Breast $\mathrm{J}$ 2013;19:49-55.

23. Sapino A, Cassoni P, Zanon E, et al. Ultrasonographicallyguided fine-needle aspiration of axillary lymph nodes: role in breast cancer management. Br J Cancer 2003;88:702-706.

24. Johnson S, Brown S, Porter G, et al. Staging primary breast cancer. Are there tumour pathological features that correlate with a false-negative axillary ultrasound? Clin Radiol 2011;66:497-499.

25. Moorman AM, Bourez RL, de Leeuw DM, Kouwenhoven EA. Pre-operative ultrasonographic evaluation of axillary lymph nodes in breast cancer patiens: for which group still of additional value and in which group cause for special attention? Ultrasound Med Biol 2015;41:2842-2848. 